$\beta$ may be obtained from Gershgorin's theorem. A method of obtaining lower bounds for the least positive eigenvalue of a certain type matrix is discussed in [5].

Bettis Atomic Power Laboratory

Westinghouse Electric Corporation

West Mifflin, Pennsylvania

1. G. Birkhoff, R. S. Varga \& D. Young, Alternating Direction Implicit Methods, Advances in Computers, Vol. 3, Academic Press, New York, 1962.

2. C. DE BOOR \& J. R. RICE, "Chebyshev approximation by a II $\left(x-r_{i}\right) /\left(x+s_{i}\right)$ and application to ADI iteration," J. Soc. Indust. Appl. Math., v. 11, 1963, pp. 159-169. MR $28 * 466$.

3. J. Dovglas, "Alternating direction methods for three space variables," Numer. Math., v. 4, 1962, pp. 41-63. MR 24 * B2122.

4. J. Douglas \& C. Pearcy, "On convergence of alternating direction procedures in the presence of singular operators," Numer. Math., v, 5, 1963, pp. 175-184. MR 27 *4384.

5. W. H. Guilinger \& R. B. Kellogg, "Eigenvalue lower bounds," submitted to the Bettis Technical Review.

6. D. W. Peaceman \& H. H. Rach Fond, Jr., "The numerical solution of parabolic and elliptic differential equations," J. Soc. Indust. Appl. Math., v. 3, 1955, pp. 28-41. MR 17, 196.

7. R. B. Smith \& J. Spanier, HOT-1: A Two Dimensional Steady-State Heat Conduction Program for the Philco-2000, WAPD-TM-465, Bettis Atomic Power Laboratory, Pittsburgh, Pa., 1964 .

8. J. Spanier \& W. H. Guilinger, "Matrix conditioning for alternating direction methods," Bettis Technical Review, WAPD-BT-29, 1963, pp. 69-79.

9. R. S. VARga, Matrix Iterative Analysis, Prentice-Hall, Englewood Cliffs, N. J., 1962. MR $28 * 1725$.

10. E. L. WAChSPRESS, "Optimum alternating-direction-implicit iteration parameter 3 for a model problem," J. Soc. Indust. Appl. Math., v. 10, 1962, pp. 339-350. MR 27 *921.

11. E. L. WACHSPRESS, "Extended application of alternating direction implicit iteration model problem theory," J.' Soc. Indust. Appl. Math., v. 11, 1963, pp. 994-1016.

12. E. L. WACHSPRESS \& G. J. HABETLER, "An alternating direction implicit iteration technique," J. Soc. Indust. Appl. Math., v. 8, 1960, pp. 403-424. MR $22 * 5132$.

\title{
An Iterative Method for Computing the Generalized Inverse of an Arbitrary Matrix
}

By Adi Ben-Israel

Abstract. The iterative process, $X_{n+1}=X_{n}\left(2 I-A X_{n}\right)$, for computing $A^{-1}$, is generalized to obtain the generalized inverse.

An iterative method for inverting a matrix, due to Schulz [1], is based on the convergence of the sequence of matrices, defined recursively by

$$
X_{n+1}=X_{n}\left(2 I-A X_{n}\right) \quad(n=0,1, \cdots)
$$

to the inverse $A^{-1}$ of $A$, whenever $X_{0}$ approximates $A^{-1}$. In this note the process (1) is generalized to yield a sequence of matrices converging to $A^{+}$, the generalized inverse of $A$ [2].

Let $A$ denote an $m \times n$ complex matrix, $A^{*}$ its conjugate transpose, $P_{R(A)}$ the perpendicular projection of $E^{m}$ on the range of $A, P_{R\left(A^{*}\right)}$ the perpendicular projection of $E^{n}$ on the range of $A^{*}$, and $A^{+}$the generalized inverse of $A$.

Theorem. The sequence of matrices defined by

$$
X_{n+1}=X_{n}\left(2 P_{R(A)}-A X_{n}\right) \quad \cdot \quad(n=0,1, \cdots),
$$

Received November 9, 1964 . 
where $X_{0}$ is an $n \times m$ complex matrix satisfying

$$
\begin{aligned}
& X_{0}=A^{*} B_{0} \text { for some nonsingular } m \times m \text { matrix } B_{0}, \\
& X_{0}=C_{0} A^{*} \text { for some nonsingular } n \times n \text { matrix } C_{0},
\end{aligned}
$$

converges to the generalized inverse $A^{+}$of $A^{1}$

Proof. As in [3], the generalized inverse $A^{+}$is characterized as the unique solution of the matrix equations,

$$
\begin{aligned}
& A X=P_{R(A)}, \\
& X A=P_{R\left(A^{*}\right)} .
\end{aligned}
$$

Thus it suffices to prove that the sequence (2) satisfies:

$$
\begin{aligned}
& \lim _{n \rightarrow \infty}\left\|A X_{n}-P_{R(A)}\right\|=0, \\
& \lim _{n \rightarrow \infty}\left\|X_{n} A-P_{R\left(A^{*}\right)}\right\|=0 .
\end{aligned}
$$

First we verify from (2), (3), (4) that

$$
\begin{aligned}
& X_{n}=A^{*} B_{n} \\
& X_{n}=C_{n} A^{*}
\end{aligned}
$$$$
(n=0,1, \cdots)
$$

(where $B_{n}, C_{n}$ are recursively computed as

$$
\begin{aligned}
& B_{n+1}=B_{n}\left(2 P_{R(A)}-A A^{*} B_{n}\right), \\
& C_{n+1}=C_{n}\left(2 P_{R\left(A^{*}\right)}-A^{*} A C_{n}\right),
\end{aligned}
$$

but are not used in the sequel).

Now, from (2),

$$
P_{R(A)}-A X_{n+1}=\left(P_{R(A)}-A X_{n}\right) P_{R(A)}-A X_{n}\left(P_{R(A)}-A X_{n}\right) ;
$$

using (12), it follows that $A X_{n} P_{R(A)}=P_{R(A)} A X_{n}$.

Therefore

$$
P_{R(A)}-A X_{n+1}=\left(P_{R(A)}-A X_{n}\right)^{2}
$$

and

$$
\left\|P_{R(A)}-A X_{n+1}\right\| \leqq\left\|P_{R(A)}-A X_{n}\right\|^{2} \quad(n=0,1, \cdots),
$$

which, by (5), proves (9).

To prove (10) we write

$$
P_{R\left(A^{*}\right)}-X_{n+1} A=P_{R\left(A^{*}\right)}-X_{n}\left(2 P_{R(A)}-A X_{n}\right) A
$$

which is rewritten, by (11), as

$$
\begin{aligned}
& \frac{P_{R\left(A^{*}\right)}-}{{ }^{1}\|\|} X_{n+1} A=P_{R\left(A^{*}\right)}-P_{R\left(A^{*}\right)} X_{n} A-X_{n} A+\left(X_{n} A\right)^{2}=\left(P_{R(A *)}-X_{n} A\right)^{2} . \\
& \text { is a multiplicative matrix norm. }
\end{aligned}
$$


Thus

$$
\left\|P_{R\left(A^{*}\right)}-X_{n+1} A\right\| \leqq\left\|P_{R\left(A^{*}\right)}-X_{n} A\right\|^{2} \quad(n=0,1, \cdots)
$$

which, by (6), proves (10).

Remarks. (i) Similarly, the sequence defined by

$$
X_{n+1}=\left(2 P_{R\left(A^{*}\right)}-X_{n} A\right) X_{n} \quad(n=0,1, \cdots),
$$

with $X_{0}$ satisfying (3), (4), (5), (6), converges to $A^{+}$.

(ii) When $A$ is nonsingular, both (2) and (16) reduce to the well-known process (1) due to Schulz [1], further studied by Dück in [4].

(iii) Conditions (5), (6) can not be weakened as shown by:

$$
A=\left(\begin{array}{ll}
1 & 0 \\
1 & 0
\end{array}\right), \quad P_{R(A)}=\left(\begin{array}{cc}
\frac{1}{2} & \frac{1}{2} \\
\frac{1}{2} & \frac{1}{2}
\end{array}\right)
$$

and, taking

$$
X_{0}=\left(\begin{array}{ll}
1 & 1 \\
0 & 0
\end{array}\right),
$$

which satisfies (3), (4) but $\left\|A X_{0}-P_{R(\Delta)}\right\|=1$ under the sum-of-squares norm.

(iv) The practical significance of the process proposed here is impaired by the need for knowledge of $P_{R(A)}$. In fact, the direct computation of $A^{+}$requires little more than the computation of $P_{R(A)}$ and of $P_{R\left(A^{*}\right)}$, and not substantially more than the computation of one alone. For any matrix $A$ can be expressed in the form $A=F R^{*}$ where the columns of $F$ are linearly independent as are those of $R$. Then, as shown by Householder in [5],

$$
P_{R(A)}=F\left(F^{*} F\right)^{-1} F^{*}
$$

and

$$
P_{R\left(A^{*}\right)}=R\left(R^{*} R\right)^{-1} R^{*}
$$

whereas

$$
A^{+}=R\left(R^{*} R\right)^{-1}\left(F^{*} F\right)^{-1} F^{*} \text {. }
$$

While only one of the projections $P_{R(A)}, P_{R\left(A^{*}\right)}$ is needed for the computation by the method proposed here, both are needed for testing (5) and (6).

(v) In the case where $A$ is of full rank, the method proposed here is applicable. For, if $\operatorname{rank} A=m, P_{R(A)}=I_{m \times m}$ and (2) reads:

$$
X_{n+1}=X_{n}\left(2 I-A X_{n}\right) \text {. }
$$

In this case, $A^{+}=A^{*}\left(A A^{*}\right)^{-1}$ and, indeed, by (11), we verify that $X_{n}=A^{*} B_{n}$, where $B_{n}$ converges to $\left(A A^{*}\right)^{-1}$.

Similarly, if $\operatorname{rank} A=n, P_{R\left(A^{*}\right)}=I_{n \times n}$ and (16) becomes

$$
X_{n+1}=\left(2 I-X_{n} A\right) X_{n} \text {. }
$$

Example. Let

$$
A=\left(\begin{array}{ccc}
1 & 0 & -1 \\
0 & 1 & 1
\end{array}\right)
$$


and take

$$
X_{0}=\frac{1}{2} A^{*}=\frac{1}{2}\left(\begin{array}{cc}
1 & 0 \\
0 & 1 \\
-1 & 1
\end{array}\right)
$$

Here, formula (17) is used to obtain:

$$
\begin{aligned}
X_{1} & =\frac{1}{2}\left(\begin{array}{cc}
1 & 0 \\
0 & 1 \\
-1 & 1
\end{array}\right)\left\{2\left(\begin{array}{ll}
1 & 0 \\
0 & 1
\end{array}\right)-\frac{1}{2}\left(\begin{array}{ccc}
1 & 0 & -1 \\
0 & 1 & 1
\end{array}\right)\left(\begin{array}{cc}
1 & 0 \\
0 & 1 \\
-1 & 1
\end{array}\right)\right\} \\
& =\frac{1}{4}\left(\begin{array}{cc}
2 & 1 \\
1 & 2 \\
-1 & 1
\end{array}\right), \\
X_{2} & =\frac{1}{16}\left(\begin{array}{cc}
10 & 5 \\
5 & 10 \\
-5 & 5
\end{array}\right), \\
X_{3} & =\frac{1}{256}\left(\begin{array}{cc}
170 & 85 \\
85 & 170 \\
-85 & 85
\end{array}\right), \quad \text { etc. }
\end{aligned}
$$

converging to:

$$
A^{+}=\frac{1}{3}\left(\begin{array}{cc}
2 & 1 \\
1 & 2 \\
-1 & 1
\end{array}\right) .
$$

Technion, Israel Institute of Technology

Haifa, Israel

1. G. Schulz, "Iterative Berechnung der reziproken Matrix," Z. Angew. Math. Mech. v. 13,1933 , pp. $57-59$.

2. R. Penrose, "A generalized inverse for matrices," Proc. Cambridge Philos. Soc., v. 51, 1955, pp. 406-413. MR 16, 1082.

3. A. Ben-IsRael \& A. Charnes, "Contributions to the theory of generalized inverses," J. Soc. Indust. Appl. Math., v. 11, 1963, pp. 667-699.

4. W. Dück, "Fehlerabschätzungen für das Iterationsverfahren von Schulz zur Bestimmung der Inversen einer Matrix," $Z$. Angew. Math. Mech., v. 40, 1960, pp. 192-194. MR 22 \#3102. 1964 .

5. A. S. Housenolder, Theory of Matrices in Numerical Analysis, Blaisdell, New York,

\title{
A Note on the Maximum Value of Determinants over the Complex Field
}

\author{
By C. H. Yang
}

The purpose of this note is to extend a theorem on determinants over the real field to the corresponding theorem over the complex field.

Theorem. Let $D(n)$ be an nth order determinant with complex numbers as its entries. Then

$$
\operatorname{Max}_{\left|a_{j k}\right| \leqq K}|D(n)|=\operatorname{Max}_{\left|a_{j k}\right|=K}|D(n)| .
$$

Received June 5, 1964. Revised December 8, 1964. 